\title{
Multi-viewpoint Smartphone AR-based Learning System for Solar Movement Observations
}

\author{
http://dx.doi.org/10.3991/ijim.v8i3.3731

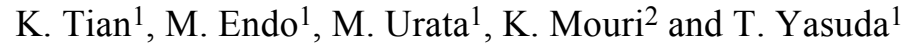 \\ ${ }^{1}$ Nagoya University, Nagoya, Japan \\ 2 Nagoya City Science Museum, Nagoya, Japan
}

\begin{abstract}
Understanding solar movement (e.g., solar diurnal motion) is difficult for those are beginning to learn about astronomy. Previous research has revealed that observation-based learning can help make astronomical phenomena clearer to understand for such learners. In this research, Smartphone Augmented Reality (AR) technology and 3D content were used to develop a multi-viewpoint Smartphone AR-based learning system (M-VSARLS) for solar movement observations that can be used in the realworld environment. The goal of this research is to assess the usefulness of the system, usability of the AR function and 3D content, and the overall effect of the system on the learner's motivation through task-based experiments with follow-up questionnaires. The results show that the M-VSARL system is effective in improving the observational skills and learning ability of learners, and in enhancing their motivation to learn about solar movement.
\end{abstract}

Index Terms-Smartphone Augmented Reality, Solar Observation, Solar Diurnal Motion, 3D Content.

\section{INTRODUCTION}

Previous research has shown that constructing a realworld-oriented learning environment is an optimal method to assist learners to grasp astronomical concepts effectively [1]. Observation-based learning is a type of real-world learning. Planetarium curators believe that the most important element in astronomy education is observing real stars from outdoor locations [2]. Based on this belief, Tian utilized smartphone augmented reality (AR) and two-dimensional (2D) content to construct an observation-based learning environment, called the multiviewpoint smartphone AR-based learning system (MVSARLS), for lunar phase observations [3]; these phase observations could be made from the earth and universe viewpoints. This research demonstrated that observationbased learning enhances the knowledge and motivation of the students learning about the lunar phases.

In addition to the lunar phases, another astronomical phenomenon that is challenging for the learners to comprehend is solar movement [4][5][6]. While considerable research has been conducted regarding solar movement [7][8][9], such studies have not yet been applied to learning in the real-world scenario. In our research, we applied the concept of M-VSARL system for solar movement observations using smartphone AR and 3D content. The proposed system can be used at any time and any location, irrespective of the weather conditions. The goal of this research is to first assess the usefulness the AR function and 3D content of this system; besides, we evaluate the system's usability and observe its effect on the motivation of the learners. A task-based experiment was conducted on 18 participants. The participants were given five solar observation tasks, which they had to perform both manually and using the M-VSARL system. After performing each task, the participants were given follow-up questionnaires that quizzed them on the usefulness of the system functions. Finally, the participants were questioned about the operational usability, and the effect that the proposed system had in motivating them to conduct solar observations. The results showed that the M-VSARL system was effective in improving the learners' observation skills and knowledge; further, it enhanced their motivation to learn about solar movement.

\section{AR IN ASTRONOMY EDUCATION}

AR is a broad concept that can be defined as "technologies that combine the real and the virtual in any location-specific way, where both real and virtual information play significant roles" [10].

AR has a wide variety of uses and can clearly demonstrate spatial concepts, temporal concepts, and contextual relationships between both real and virtual objects. The main advantages of using virtual objects are as follows: virtual objects can be animated; they respond to user actions; they are not constrained by costs, time, and any other practical or physical limitations that apply on real objects. These factors make AR a powerful educational tool [11]. For example, Shelton explored the use of AR in teaching undergraduates about earth-sun relationships in terms of axial tilt and solstices, and found that AR is useful for teaching subject content that students cannot possibly experience firsthand in the real world [12]. Live Solar System (LSS) is an AR-book based educational tool created with the objective of helping students learn astronomy. LSS incorporates the Tangible AR approach in its design that allows users to interact naturally and intuitively with the system. This novel approach provides learners a new and more engaging learning experience [13].

Interest in mobile $\mathrm{AR}$ applications has grown concomitant with the dramatic increase in the use of smartphones over the past few years. Present-day smartphones and tablets, which integrate fast processors with graphics hardware, large touch screens, and onboard sensors (camera, GPS, compass, and accelerometers), are gadgets that can facilitate ideal indoor as well as outdoor AR experiences. Researchers and commercial developers have used these platforms to develop educational applications that provide novel learning experiences [14]. There are many popular AR astronomy applications that 
can be applied to outdoor use, such as Google Sky Map, Star Walk, and Stellarium Mobile. These applications utilize the Global Positioning System (GPS) in smartphones and other sensors to locate celestial bodies such as the sun and the stars. These applications name the particular star that is being viewed through the user's phone. Their primary purpose is to locate the stars and planets in the sky. However, they are not designed for learning or teaching about solar movement; for example, they do not have dynamic lines of latitude and longitude to help learners study the azimuth and angle of elevation of the sun in the sky. In addition, these applications cannot address the solar motion learning goals outlined in science education. For example, they have no functions showing the orbit of solar diurnal motions for different times of the year and at different locations in the same AR view. Further, these applications lack a function explaining the mechanism of solar motion because it relates to everyday observations of solar movement at different times and locations.

The M-VSARL system assists learners in observing and studying solar movement, and achieves the educational goals of solar movement in a real-world environment.

\section{M-VSARL SYSTEM}

This section describes the learning goal of the system, the system design, the system interface, and its functionality.

\section{A. Learning Goal of Solar Observations}

The proposed system was developed as an educational tool for solar observations. The learning functions of the system are based on the learning indicators in the science course titled Solar Movement. We set the learning goals of solar observations for the M-VARML system as follows:

(1) Observe the sun each day to determine its azimuth and altitude, and witness it rising in the east and setting in the west.

(2) Observe the solar diurnal motion orbit, noon altitude, and length of daytime during the vernal equinox, summer solstice, autumn equinox, and winter solstice.

(3) Observe the solar diurnal motion orbit, noon altitude, and length of daytime at the same time at different locations, namely, from Japan, the Equator, and the North Pole.

(4) Understand the positional relationship between the sun and earth to obtain a view of the mechanism of solar motion at different times and locations throughout the universe.

\section{B. System Outline}

We have proposed helping learners understand astronomy topics, such as lunar phase from multiple viewpoints - namely, the geocentric model and the heliocentric model viewpoint [3]. Therefore, the MVSARL system enables learners to observe the sun's movement from the viewpoint of the earth, and at the same time, understand the relationship between the sun and the earth from the sun-center viewpoint of the universe when studying the mechanism of solar movement. An outline of the system is shown in Fig. 1.
The M-VSARL system has two main viewpoints: an AR viewpoint and a universe viewpoint. Learners can switch between the two to observe solar activities and thereby achieve the previously stated goal of learning about solar movement. The following system specifications were used to facilitate a real-world learning environment in order to achieve the specified learning goal (Refer Section III-A):

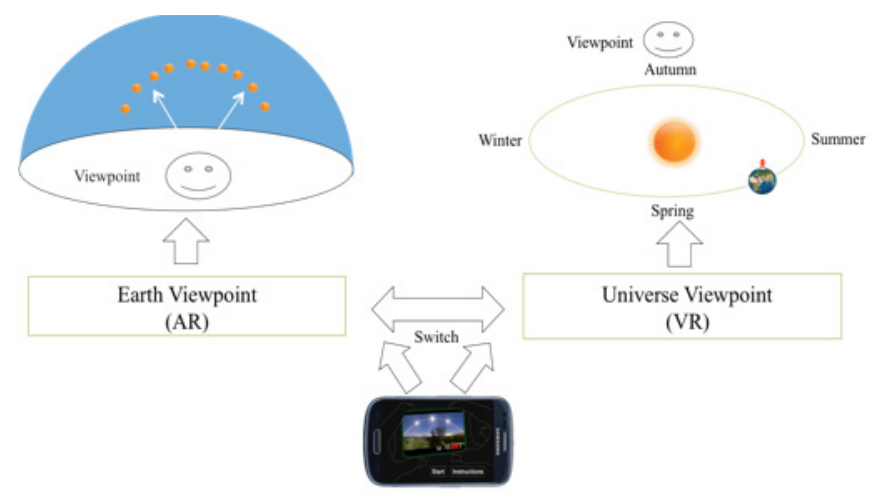

Figure 1. Outline of the M-VARML system

(a) The azimuth or the altitude of the sun can be observed in the actual sky using the AR view function.

(b) The solar orbit can be observed in the real sky between 1:00 a.m. and 12:00 p.m. using the OneDay function.

(c) Solar diurnal motion orbit during the vernal equinox, summer solstice, autumn equinox, and winter solstice can be observed using the ShowSeasonNoonAltitude function.

(d) Solar diurnal motion orbit at the Equator and North Pole can be observed using the ShowLocationNoonAltitude function.

(e) The relationship between the sun and the earth, i.e., the mechanism of solar diurnal motion can be understood using the Universe View function.

Among these specifications, (a) and (b) help achieve learning goal (1), (c) helps achieve learning goal (2), (b) and (d) help achieve learning goal (3), and (e) helps achieve learning goal (4).

\section{Astronomy Simulation Model}

The M-VARML system is based on the astronomy model in which the earth, while rotating on its own axis, revolves around the sun. Our research group has been focusing on the simulation of astronomy phenomena for a considerably long time. Based on the astronomy simulation model shown in Fig. 2, several astronomy education systems [15][16] have been developed. In this research, we employed this astronomy simulation model to develop the M-VARML system for supporting solar movement observations from multiple viewpoints.

\section{AR View Function}

The M-VSARL system shows virtual images of the sun overlaid onto a live video camera background, making the virtual sun images appear to exist in the real world. To visualize the sun correctly in AR view, real-time data are obtained from the smartphone through its built-in sensors. Subsequently, these data are combined with the astronomy 


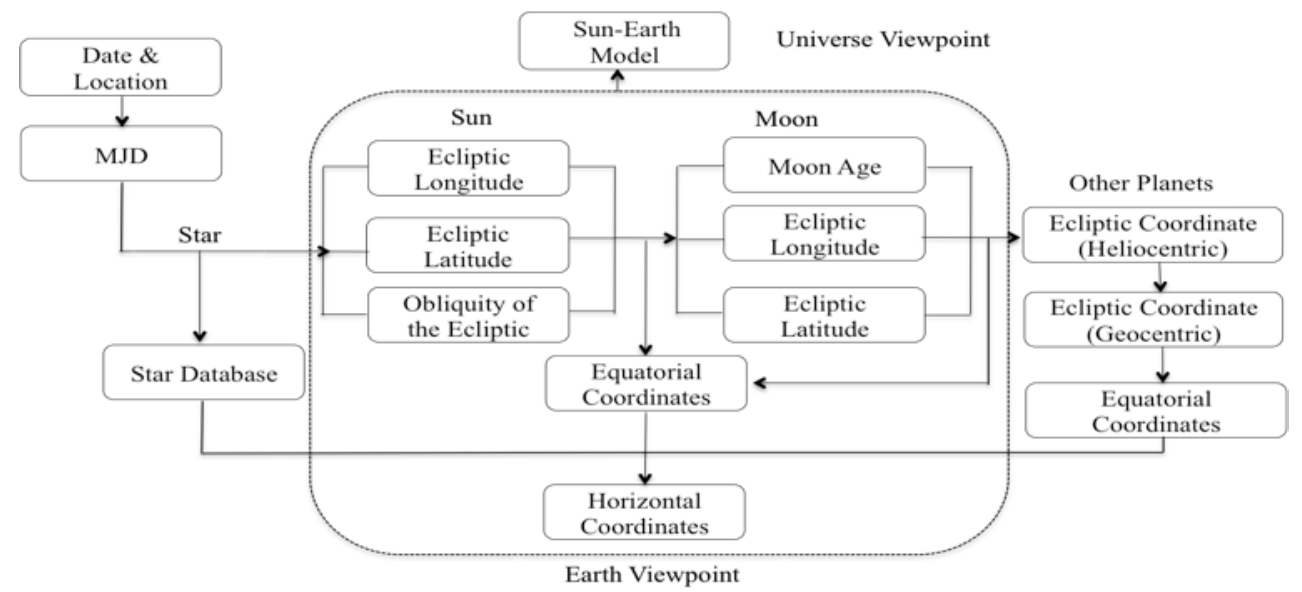

Figure 2. Astronomy simulation model

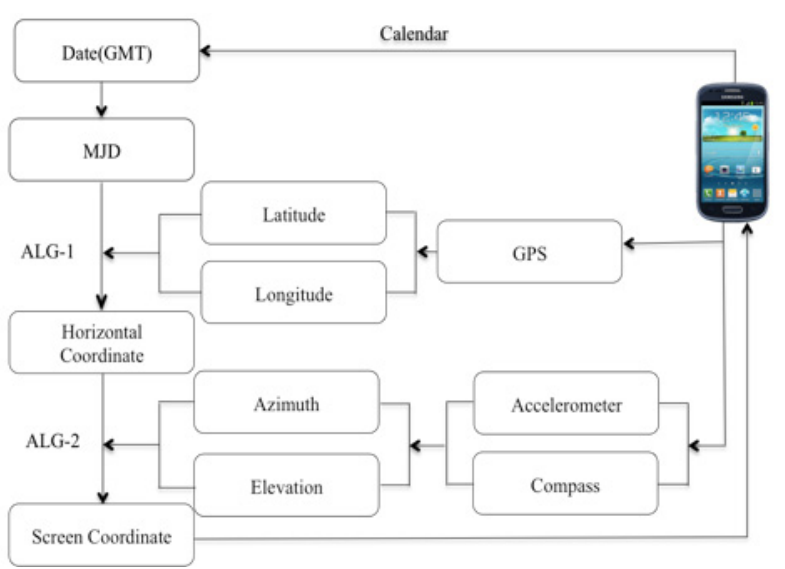

Figure 3. Procedure for calculating the sun's position in AR view

simulation model (see Fig. 2) to calculate the screen coordinates of the visual sun in AR view and correctly register it in the real sky, which the users can observe.

The procedure for calculating the position of the sun is outlined in Fig. 3. First, the date and time are obtained in real-time from the calendar of the smartphone and are used to calculate the Modified Julian Day (MJD) [17]. Subsequently, longitude and latitude data are obtained using the GPS sensor, which is responsible for tracking the geographical position of the smartphone. Combining these data with the horizontal coordinates algorithm (ALG-1), the azimuth and elevation of the sun can be computed in real time. The azimuth and elevation data are the horizontal coordinate data of the sun in the sky. These data are used to derive the screen coordinates of the sun and register the sun in AR view at a location corresponding to the actual location of the sun in the sky. The accelerometer and compass sensors measure the viewing direction and elevation angle of the smartphone while observing the sun in the sky. Based on this tracking information, we use the screen projection algorithm (ALG-2) to convert the horizontal coordinates into screen coordinates to situate the sun in AR view. Following this procedure, the virtual sun is shown in AR view and is appropriately mapped to the real sun in the sky.

The complete AR view interface of the M-VSARL system is shown in Fig. 4. The data part shows the orientation, angle of elevation of the mobile device, the GPS location, date, time, and azimuth and elevation of the

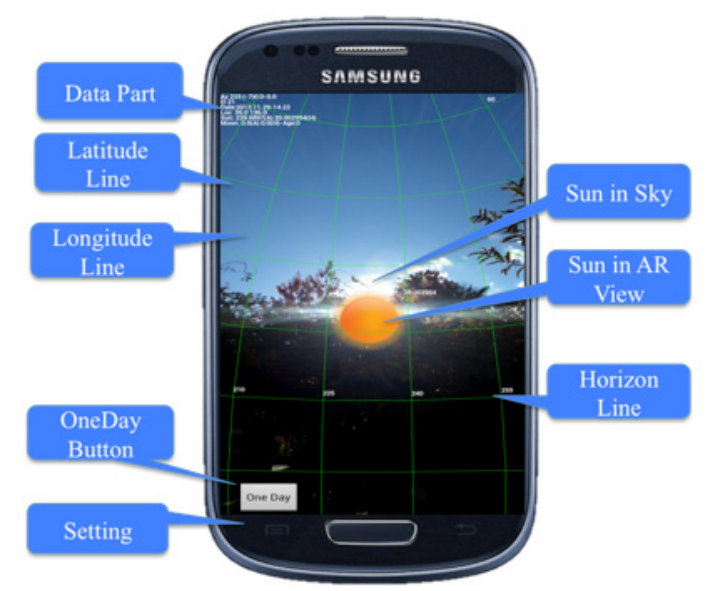

Figure 4. AR view interface

sun. The horizontal line is the zero degree latitude line, and indicates the horizon in the real world. The notations $\mathrm{N}, \mathrm{E}, \mathrm{S}$, and $\mathrm{W}$ (corresponding to north, east, south, and west, respectively) indicate the direction the smartphone is pointing towards in the real world. These directions, in combination with the lines of latitude and longitude, can help learners locate and observe the sun. In addition, learners can modify the settings such as the degrees of latitude and longitude, the size of the text, and the color from the setting interface.

The OneDay functions in the AR view meet the specification-learning environment (b), and its interface is shown in Fig.5. Further more, the ShowSeasonNoonAltitude function in AR view satisfies specification-learning environment (c), and its interface is shown in Fig. 6. Learners can observe the solar orbit, noon altitude of the sun, and the length of the daytime during the vernal equinox, summer solstice, autumn equinox, and winter solstice. For example, the highest solar altitude among the four solar terms occurs during the summer solstice, as indicated by the red line. In addition, the ShowLocationNoonAltitude in the AR view meets specification-learning environment (d), and the interface as shown in Fig. 7. Learners can observe the solar orbit, noon altitude of the sun, daytime length, and the rising and setting of the sun from three locations. For example, in this view, learners can intuitively grasp that the solar diurnal motion orbit at the North Pole is parallel to the equatorial line, i.e., the yellow line shown in Fig. 7. 


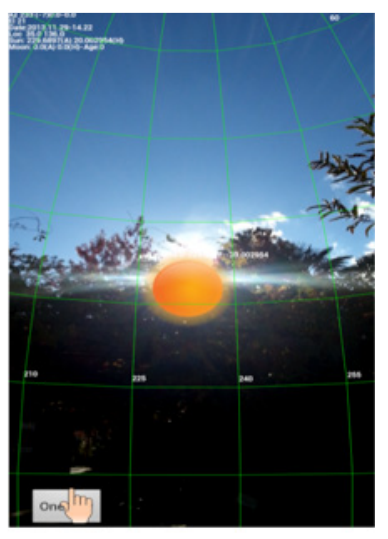

(a)

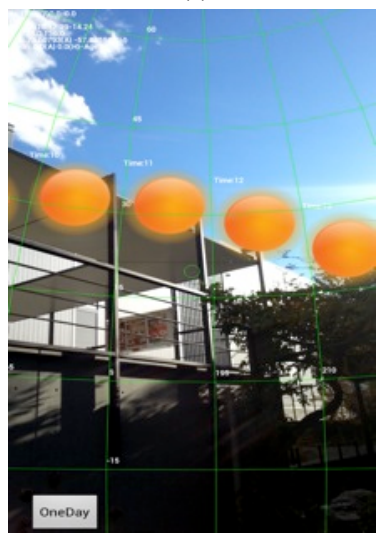

(c)

Figure 5. One day orbit of the sun in AR view

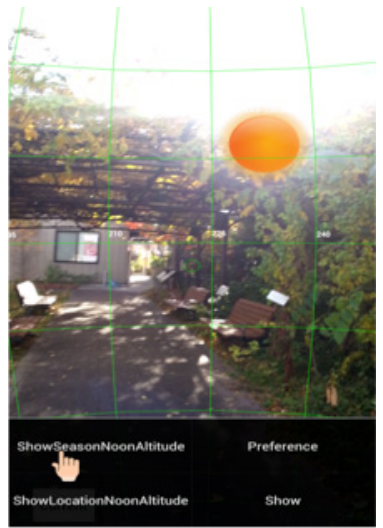

(a)

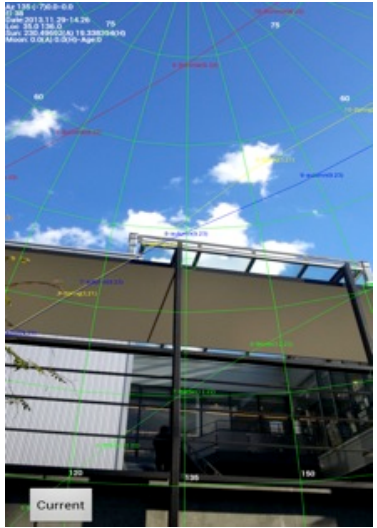

(c)

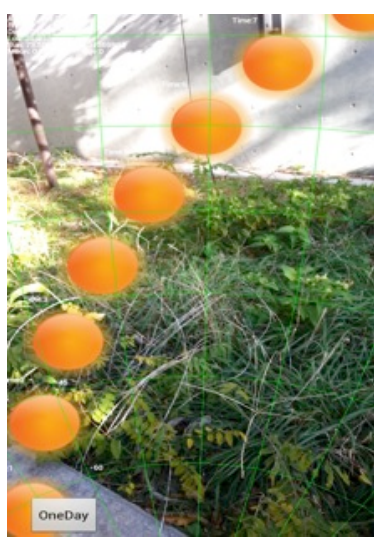

(b)

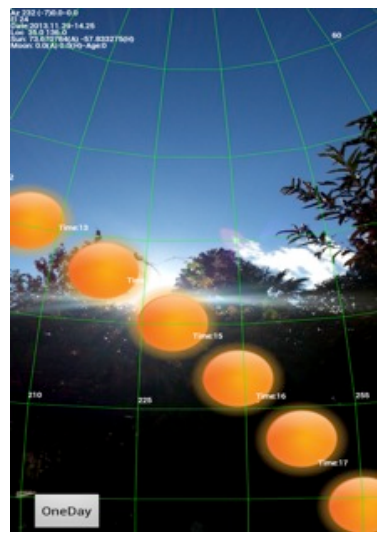

(d)

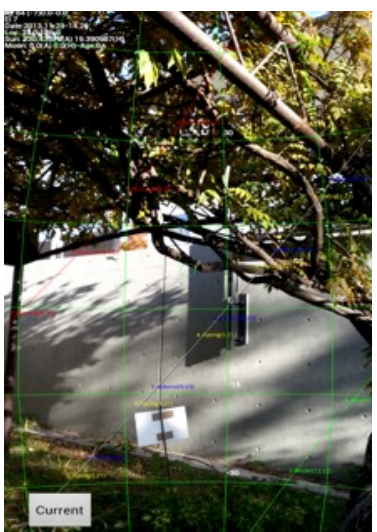

(b)

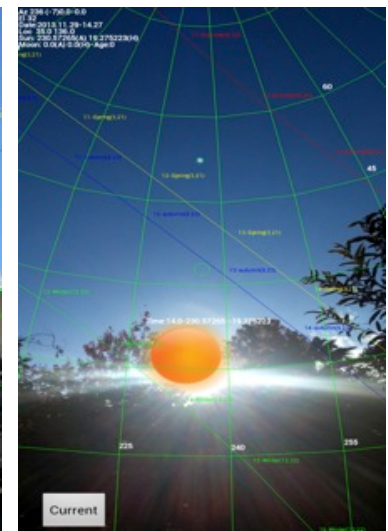

(d)
Figure 6. Solar diurnal motion orbit during four solar terms

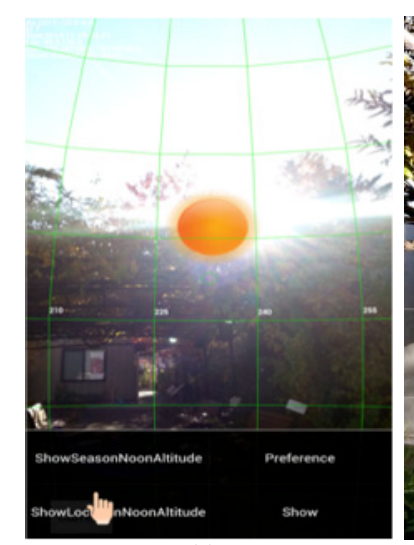

(a)

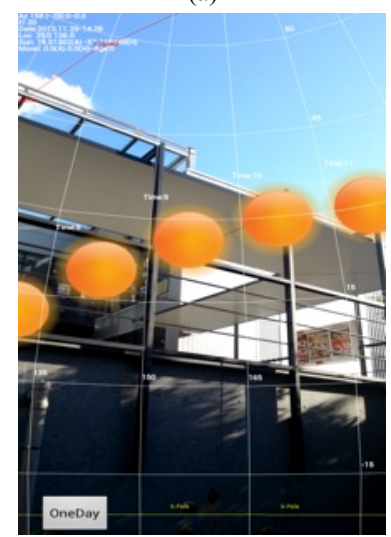

(c)

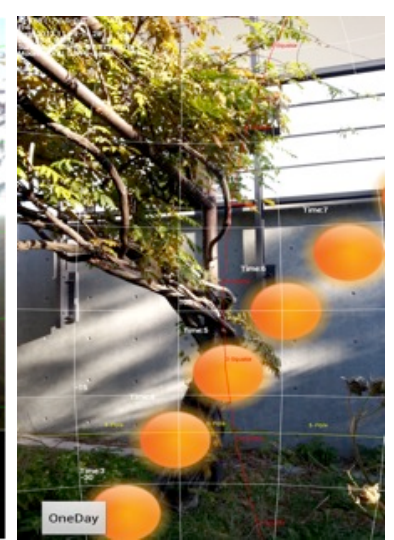

(b)

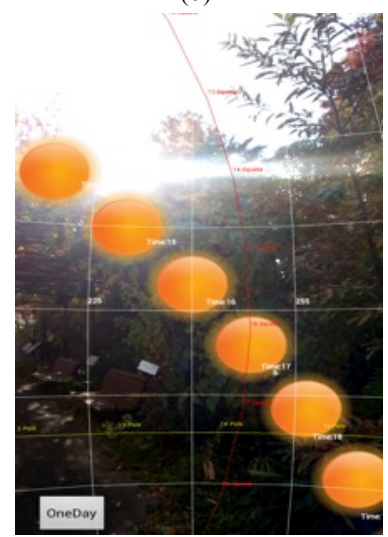

(d)

Figure 7. Solar diurnal motion orbit from Japan, the Equator, and the North Pole.

Through the AR view function, learners can not only construct a visual feel for solar observations of the sky, but can also complete the learning goal of solar observations from a earth viewpoint at any location and at any time regardless of the weather restrictions.

\section{E. Universe View Function}

In a previous study [3], we utilized 2D content to develop a universe view. However, during the experiment, most of the participants considered that it was better to use a $3 \mathrm{D}$ space to show the relationship of the sun, earth, and moon. Further, previous research has shown that 3D modeling can help students overcome their misconceptions of astronomical concepts, such as the solar system [18]. Therefore, in this research, we used Android OpenGL to develop a real-time 3D sun-earth movement simulator to help learners understand the earth's revolution and rotation from a universe view. When the user touches the current sun image in AR view, the user can see an item called the Mechanism. Selecting this item lands the user in the universe view interface, which is shown in Fig. 8.

During the year, the seasons change from spring to summer, autumn, and winter. Thus, from the universe view, we can visualize the four key stages of the earth's revolution in orbit, namely, spring (vernal equinox), summer (summer solstice), autumn (autumn equinox), and winter (winter solstice). In fact, the solar diurnal motion orbit in one day is due to the earth's rotation and tilt. Further, the altitude of the sun at noon differs during the four seasons because of the earth's rotation and because the earth completes a revolution around the sun through the year. Fig. 8 illustrates the earth revolving around the- 


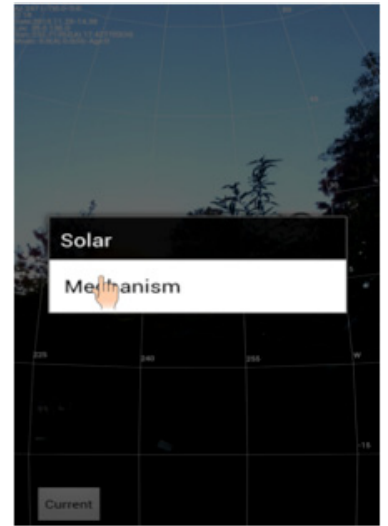

(a)

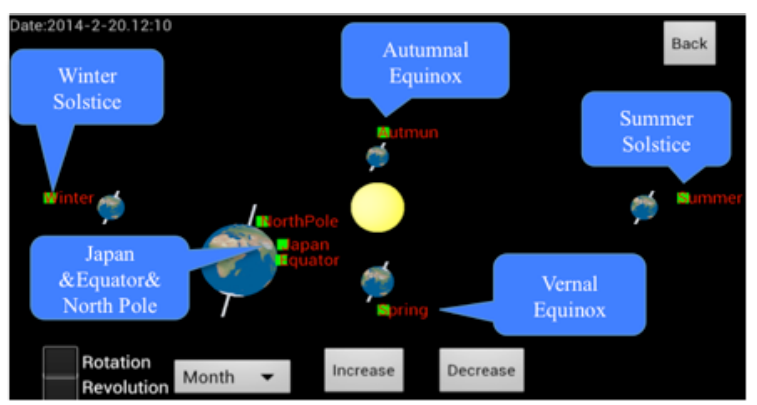

(b)

Figure 8. Entering universe view
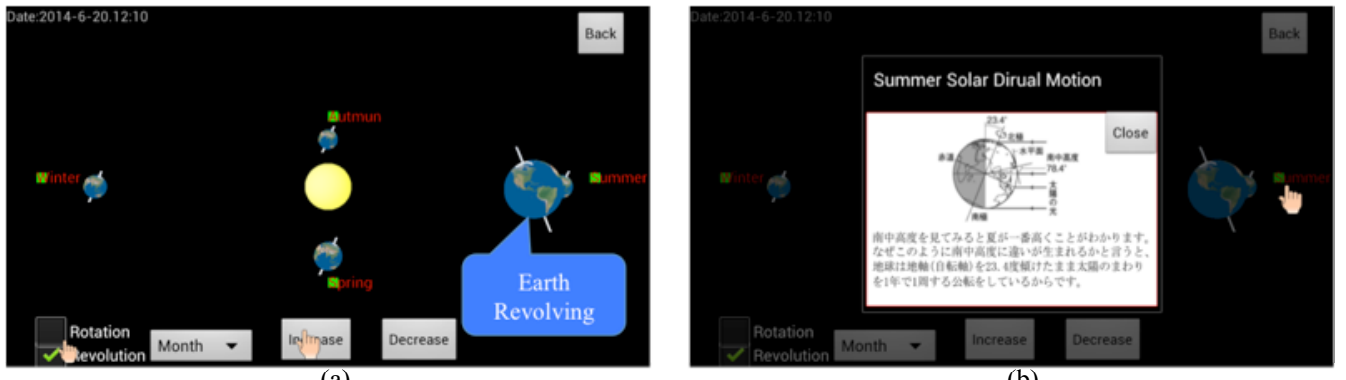

(b)

Figure 9. Revolving of the earth

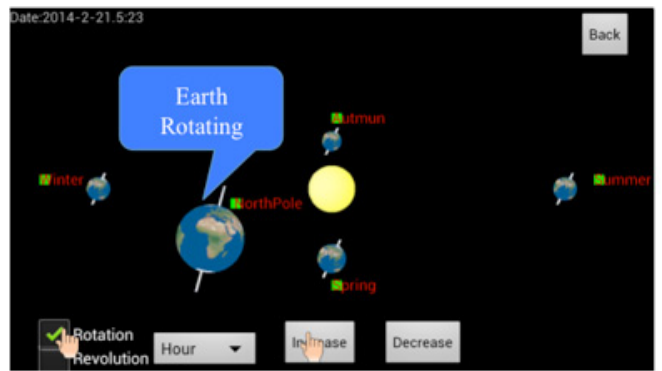

(a)

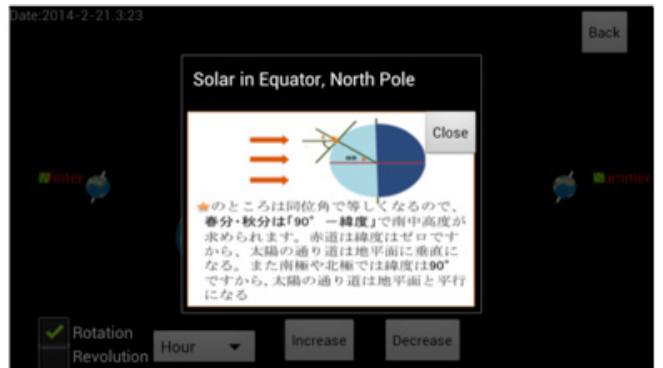

(b)

Figure 10. Rotation of the earth

sun while rotating on its axis, causing days and nights. The earth requires approximately 365 days to revolve around the sun. During the year, the seasons change from spring to summer, autumn, and winter. Thus, from the universe view, we can visualize the four key stages of the earth's revolution in orbit, namely, spring (vernal equinox), summer (summer solstice), autumn (autumn equinox), and winter (winter solstice). Using this view, learners can understand the position and relationship between the sun and the earth in orbit. We also provide users with an interactive function to observe the relationship between the sun and the earth, and the earth's rotation and revolution. Learners can choose the revolution function, and click on the increase and decrease buttons to make the earth revolve around the sun, as shown in Fig. 9. Learners can thus observe the earth revolving around the sun during the year. To assist learners to understand the mechanism of solar motion during the four seasons, learners can touch on the text describing each season; for example, on touching summer, an image and text describing the mechanism of solar diurnal motion during summer will be displayed. As with the revolution function, learners can choose the rotation option to experience the earth rotating throughout the day, and after touching the location text in universe view, as shown in Fig. 10, an image and text appear describing why different solar diurnal motion orbits occur in Japan, the Equator, and the North Pole.

The universe view function satisfies the stated learning environment specification (e) by improving the user's knowledge regarding the mechanism of solar diurnal motions

\section{SYSTEM IMPLEMENTATION}

We developed the M-VSARL system on the Google Android operating system using the Eclipse toolset, ADT, and the Android 2.1 Software Development Kit (SDK). We utilized the Java programming language and developed the 3D models of the sun and the earth using the Android OpenGL API. The resulting application is supported by a range of smartphone and tablet devices that are compatible with Android 2.1 or higher, and have the required sensors (i.e., a camera, GPS, electronic compass, and accelerometer). We implemented the M-VSARL system on a Samsung Galaxy S3 Android smartphone. 


\section{EXPERIMENT DESIGN}

A task-based experiment was conducted with 18 participants to confirm the usefulness and usability of the system. The 18 participants (three female, fifteen male) were undergraduate and graduate students with majors unrelated to astronomy, and were verified to have an interest in solar movement observations. The participants were requested to perform a series of solar observational tasks during different times in a day, at various locations. Some example observational tasks are shown in Fig. 11.

There were a total of five tasks, which were based on the aforementioned learning goals of solar observation (see Section II-A). The tasks are listed in Table I. After each task was completed, the participants were given follow-up questions seeking the usefulness of the MVARML system corresponding to the task, as shown in Fig. 12. These questions are listed in Table II. The purpose of the five tasks was to evaluate the usefulness of the proposed system. The chief purpose of each task was to evaluate the usefulness of each system function. As an example, consider task 2 that was conducted to evaluate the usefulness of the OneDay function that helps learners achieve learning goal (2).

After completing all five tasks and the corresponding questions regarding the usefulness of the proposed system, participants were given six questions and a free-response item on its usability and their overall satisfaction. These questions are listed in Table III. All surveys used a fivepoint Likert-scale, the responses to which were coded as 1 (strongly disagree) through 5 (strongly agree). We will compute the mean value of each questionnaire item regarding the system's usefulness, usability, and user satisfaction.

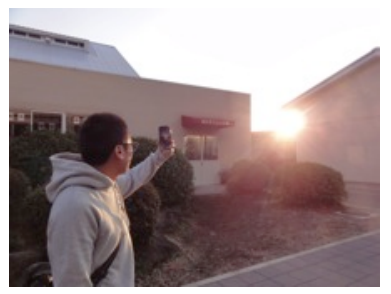

(a)

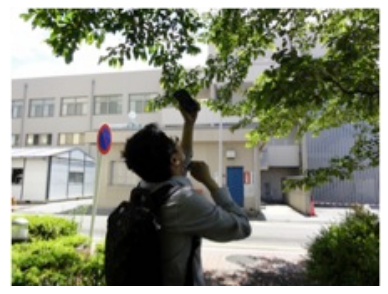

(b)
Figure 11. Experimental situations

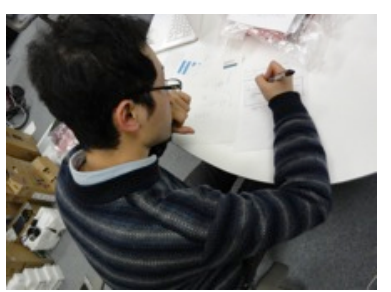

(a)

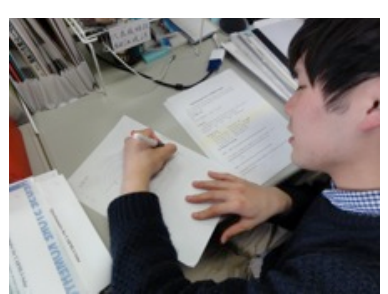

(b)
Figure 12. Completing the task scene

\section{EXPERIMENT RESULTS}

\section{A. System Usefulness}

The results of the survey pertaining to the usefulness of the proposed system during the task stage are shown in Table IV. The mean of questionnaire item Q1 was 4.50,
TABLE I.

LIST OF EXPERIMENT TASKS

\begin{tabular}{|c|c|}
\hline No. Items & Contents \\
\hline Task1 & $\begin{array}{l}\text { First, participants locate and observe the sun in real } \\
\text { time to guess its azimuth and elevation angle } \\
\text { without using the proposed system. Next, the users } \\
\text { state or write down the azimuth and elevation angle } \\
\text { of the sun. Subsequently, they choose the function } \\
\text { showing the latitude and longitude lines in AR } \\
\text { view, and use the system to find and observe the } \\
\text { azimuth and elevation angle of the sun in the sky. }\end{array}$ \\
\hline Task2 & $\begin{array}{l}\text { First, participants guess the one-day solar orbit and } \\
\text { use their fingers to draw the line of this orbit in the } \\
\text { sky, or draw it on paper with a pen. To confirm } \\
\text { their drawing, users use the OneDay function to } \\
\text { observe the one-day solar orbit in AR view. }\end{array}$ \\
\hline Task3 & $\begin{array}{l}\text { First, the participants observe the sun in real time, } \\
\text { and guess solar diurnal motion orbit during the } \\
\text { vernal equinox, summer solstice, autumn equinox, } \\
\text { and winter solstice. Subsequently, they draw a line } \\
\text { of the one-day solar orbit (vernal equinox, summer } \\
\text { solstice, autumn equinox, and winter solstice) on } \\
\text { paper with a pen. To confirm users drawing, the } \\
\text { participants use the ShowSeasonNoonAltitude } \\
\text { function to observe the solar diurnal motion orbit } \\
\text { during the four seasons in AR view. }\end{array}$ \\
\hline Task4 & $\begin{array}{l}\text { First, the participants observe the sun, and estimate } \\
\text { the solar diurnal motion orbit from Japan, the } \\
\text { Equator, and the North Pole concurrently (vernal } \\
\text { equinox). Subsequently, the participants draw the } \\
\text { solar diurnal motion orbit from Japan, the Equator, } \\
\text { and the North Pole on paper with a pen. To confirm } \\
\text { users drawing, the participants can click on the } \\
\text { ShowLocationNoonAltitude and One Day functions } \\
\text { to observe the solar diurnal motion orbit from the } \\
\text { three locations in AR view. }\end{array}$ \\
\hline Task5 & $\begin{array}{l}\text { First, participants according to the solar to guess } \\
\text { the relationship between the sun and the earth in the } \\
\text { universe view; they draw this relationship on paper } \\
\text { with a pen, and describe the mechanism of solar } \\
\text { diurnal motion orbit for the different seasons and } \\
\text { locations. Subsequently, they click on the solar } \\
\text { image to enter universe view to confirm their } \\
\text { answers. }\end{array}$ \\
\hline
\end{tabular}

TABLE II.

USEFULNESS QUESTIONNAIRE

\begin{tabular}{|c|l|}
\hline No. Items & \multicolumn{1}{c|}{ Contents } \\
\hline Q1 & $\begin{array}{l}\text { The latitude and longitude lines of the system are } \\
\text { helpful for understanding the azimuth and elevation } \\
\text { angle of the sun in the sky in real time. }\end{array}$ \\
\hline Q2 & $\begin{array}{l}\text { The OneDay function of the system is helpful for } \\
\text { observing the movement orbit of one-day solar } \\
\text { across the starry sky. }\end{array}$ \\
\hline Q3 & $\begin{array}{l}\text { The ShowSeasonNoonAltitude function of the } \\
\text { system is helpful for observing the solar diurnal } \\
\text { motion orbit, noon altitude, and daytime length } \\
\text { during the vernal equinox, summer solstice, autumn } \\
\text { equinox, and winter solstice. }\end{array}$ \\
\hline Q4 & $\begin{array}{l}\text { The ShowLocationNoonAltitude function of the } \\
\text { system is helpful in observing the solar diurnal } \\
\text { motion orbit, daytime length, and noon altitude } \\
\text { from Japan, the Equator, and the North Pole } \\
\text { simultaneously. }\end{array}$ \\
\hline Q5 & $\begin{array}{l}\text { The Universe View function of the system is helpful } \\
\text { in understanding the sun-earth relationship, as well } \\
\text { as the mechanism of solar diurnal motion for } \\
\text { different seasons and locations from the universe } \\
\text { viewpoint. }\end{array}$ \\
\hline
\end{tabular}


TABLE III.

USABILITY AND SATISFACTION QUESTIONNAIRE

\begin{tabular}{|c|l|}
\hline No. Items & Contents \\
\hline Q6 & It is easy for me to operate the system. \\
\hline Q7 & The interface was clear and easy to understand \\
\hline Q8 & $\begin{array}{l}\text { The response time of system is short when you are } \\
\text { operating the system. }\end{array}$ \\
\hline Q9 & $\begin{array}{l}\text { It is interesting for me to use the system to observe } \\
\text { solar movement }\end{array}$ \\
\hline Q10 & $\begin{array}{l}\text { The system is helpful to me in enhancing my } \\
\text { motivation on solar movement. }\end{array}$ \\
\hline Q11 & $\begin{array}{l}\text { I would like to use the same kind of system for } \\
\text { astronomy learning in the future. }\end{array}$ \\
\hline
\end{tabular}

showing that the users deemed the latitude and longitude line in AR view to be helpful in observing the azimuth and elevation angle of the sun in the sky. During task 1, users agreed that the latitude and longitude lines were helpful in constructing a visual feel of the azimuth and elevation angle in the real sky. Therefore, for task 2, most of the participants were able to use their fingers to draw the correct solar orbit in the sky after they determined the east and west directions in the real world, and they confirmed that the OneDay function is very useful in observing the sunrise and sunset in a day in the sky (Q2). The mean of Q3 is 4.61, proving that the participants conclusively agreed that the ShowSeasonNoonAltitude function was helpful in observing the solar diurnal motion orbit, daytime length, and noon altitude during the vernal equinox, summer solstice, autumn equinox, and winter solstice. Further, some users pointed out that this function helped them resolve some misconceptions, such as their previous belief that sun rises from due east during all four solar terms.

Similar misconceptions were discovered while performing task 4, which only five users could complete. Here, the participants conclusively felt that the ShowLocationNoonAltitude function was helpful in observing the solar diurnal motion orbit, daytime length, and noon altitude from Japan, the Equator, and the North Pole simultaneously, as is evident from the mean of 4.56 for Q4. All the questionnaire items (Q1, Q2, Q3, and Q4) regarding the usefulness of the $A R$ view had a mean of above 4.28, which indicates that the AR view is very helpful to learners in observing the solar movement, and provides a visual real-world learning experience. In addition, six of the participants successfully completed task 5, and the users felt that the Universe View function was helpful in understanding the relationship between the sun and the earth in universe view, as well as in comprehending the mechanism of solar diurnal motion through the images and text for different times and locations from the universe viewpoint.

Based on this analysis and the experiment results, we can draw the conclusion that the M-VSARL system is very useful for learners in observing solar movement in a real-world environment.

TABLE IV.

USEFULNESS QUESTIONNAIRE RESULTS

\begin{tabular}{|c|c|c|c|c|c|c|}
\hline No. Items & $\mathbf{1}$ & $\mathbf{2}$ & $\mathbf{3}$ & $\mathbf{4}$ & $\mathbf{5}$ & Mean \\
\hline Q1 & 0 & 0 & 0 & 9 & 9 & 4.50 \\
\hline Q2 & 0 & 0 & 4 & 5 & 9 & 4.28 \\
\hline Q3 & 0 & 0 & 1 & 5 & 12 & 4.61 \\
\hline Q4 & 0 & 0 & 1 & 6 & 11 & 4.56 \\
\hline Q5 & 0 & 0 & 3 & 8 & 7 & 4.22 \\
\hline
\end{tabular}

TABLE V.

USABILITY AND SATISFACTION QUESTIONNAIRE RESULTS

\begin{tabular}{|c|c|c|c|c|c|c|}
\hline No. Items & $\mathbf{1}$ & $\mathbf{2}$ & $\mathbf{3}$ & $\mathbf{4}$ & $\mathbf{5}$ & Mean \\
\hline Q6 & 0 & 0 & 2 & 6 & 10 & 4.44 \\
\hline Q7 & 0 & 0 & 2 & 7 & 9 & 4.39 \\
\hline Q8 & 0 & 1 & 2 & 5 & 10 & 4.33 \\
\hline Q9 & 0 & 0 & & 8 & 10 & 4.56 \\
\hline Q10 & 0 & 0 & 2 & 2 & 14 & 4.67 \\
\hline Q11 & 0 & 0 & 2 & 5 & 11 & 4.50 \\
\hline
\end{tabular}

\section{B. System Usability}

In terms of the interface design, most of the users agreed that the system was easy to operate, with a mean value of 4.44 for Q6, as shown in Table V. The users were able to operate the system and complete their solar observation tasks. In addition, the interface was clear and easy for the users to understand with a mean value of 4.39 for Q7. However, the participants did report that the data in AR view were hard to understand because no explanation of these data was given before conducting the task. In addition, the mean of 4.33 for Q8 showed that the response time when operating the system was brief. There were also some response problems in Universe View because the area of the touch event is narrow. During our interviews with the participants, the users reported that the size of the text should be larger and that a different set of colors would make the interface easier to understand.

\section{System Satisfaction}

In addition, the participants appeared to be highly interested in using the system to observe solar movement, with a mean value of 4.56 for Q9. Almost all of the participants agreed that they originally did not have a strong intention to observe the solar movement during the daytime. However, after using the system, their motivation to conduct solar observations was enhanced, which is reflected in the mean value of 4.67 for Q10. In the future, the participants stated that they would prefer to use the same kind of AR learning system to observe other astronomy phenomena, such as season constellation, which was proved by a mean value of 4.50 for Q11. In other words, the participants were highly satisfied with the M-VSARL system, and believed that such a system can increase their interest in astronomy.

In the questionnaire, we also requested the participants to share their opinions regarding the proposed system candidly. Two of the participants mentioned that it would be better if we highlighted the horizon line, and one user suggested that we use arrows as guides for locating the sun.

\section{CONCLUSION}

In this paper, we described the utilization of smartphone AR technology and $3 \mathrm{D}$ content in the construction of a multi-viewpoint observation environment for learners observing solar motion in a real-world environment. The system has an AR view that can be used to observe the azimuth, angle of elevation, and solar diurnal motion orbits during the four seasons and at different locations. Further, it has a universe view that can be used to understand the solar motion mechanism from the universe viewpoint to assist learners with solar observations. 
We assessed the usefulness, usability, and motivational influence of the proposed M-VSARL system through task-based experiments. The results of our experiments revealed that each function of the system is very usable and useful. The M-VSARL system allowed learners to improve their knowledge and complete a set of solar observation learning goals. Further, it enhanced the motivation levels of the learners.

For future work, we plan to improve the interface design of the system to ensure that even the learners with limited knowledge can use it. Moreover, we intend to add additional features such as the ability to provide astronomy news regarding solar movement.

\section{ACKNOWLEDGMENT}

The M-VSARL system for solar observation developed through this research is an important part of a project titled Astronomy Education Platform for use in Smartphone Augmented Reality Technology. This project was supported by the Grants-in-Aid for Scientific Research Japan (No. 25280131) and NTT Docomo Tokai in Japan. In addition, we would like to thank Mr. Tom Kabara of Mei Writing in Nagoya University for his patient guidance in writing this paper.

\section{REFERENCES}

[1] M. Soga, M. Ohama, Y. Ehara, and M. Miwa, "Real-World Oriented Mobile Constellation Learning Environment Using Gaze Pointing," IEICE Transactions on Information and Systems, vol. 94, no. 4, pp. 763-771, 2011. http://dx.doi.org/10.1587/ transinf.E94.D.763

[2] M. Kondo, M. Mouri, and Y. Yausda, "A Multiplatform Content Management System for Curators to Provide Teaching Materials in Astronomy Education," Journal of Theoretical and Applied Information Technology, vol. 4, no. 9, pp. 874-881, 2005.

[3] K. Tian, M. Endo, M. Urata, K. Mouri, and T. Yasuda, "Lunar Observation Support System Using Smartphone AR for Astronomy Education," International Journal of Interactive Mobile Technologies, vol. 8, no. 1, pp. 32-39, 2014 http://dx.doi.org/10.3991/ijim.v8i1.3457

[4] National Institute for Education Policy Research. A Survey about the Elementary School and Junior High School Curriculum Situation-Science Education in 2003, http://www.nier.go.jp/kaihatsu/katei_h15/H15/0300104003000700 4.pdf

[5] A. Ito, M. Chida, and H. Tabara, "Variation of Astronomical Knowledge of University Students Revealed from Investigations carried out in 1976 and 2006," Bulletin of Integrated Research Center for Educational Practice Faculty of Education, Utsunomiya University, vol. 30, no. 7, pp. 473-482, 2007.

[6] I N. Diakidoy and P. Kendeou, "Facilitating Conceptual Change in Astronomy: A Comparison of the Effectiveness of Two Instructional Approaches," Learning and Instruction, vol.11, no.1, pp.1-20, 2001. http://dx.doi.org/10.1016/S0959-4752(00)00011-6

[7] S M. Wang, "Study on Building a Virtual Reality Model for Observing the Movements of the Moon and the Sun for Elementary Schools and Junior High Schools," M.S. Thesis, National University of Tainan, China, 2007.

[8] J. Yamashita, H. Kuzuoka, C. Fujimon, and M. Hirose, "Tangible Avatar and Tangible Earth: A Novel Interface for Astronomy Education," Proceedings of CHI 2007 Human Factors in Computing Systems, pp. 2777-2782, April 30 - May 03, 2007.

[9] T. Takata, K. Saito, S. Kadowaki, E. Kuwabara, T. Aida, T. Miyaji, and T. Chihima, "Space Experiments in Classrooms-10:
Development of Teaching Materials of Solar Diurnal Motions Taken by the Whole Sky Cameras," Bulletins of Miyagi University of Education, vol. 45, pp. 71-79, 2011.

[10] E. Klopfer and K. Squire, "Environmental Detectives-the Development of an Augmented Reality Platform for Environmental Simulations," Educational Technology Research and Development, vol. 56, no. 2, pp. 203-228, 2008. http://dx.doi.org/10.1007/s11423-007-9037-6

[11] E. Woods, M. Billinghurst, J. Looser, G. Aldridge, D. Brown, B. Garrie, and C. Nelles, "Augmenting the Science Centre and Museum Experience," In 2nd International conference on Computer Graphics and Interactive Techniques in Australia and Southeast Asia, Suntec City, Singapore, pp. 230-236, June 15-18, 2004.

[12] B E. Shelton and N. Hedley, "Using Augmented Reality for Teaching Earth-Sun Relationship to Undergraduate Geography Students," The First IEEE International Augmented Reality Toolkit Workshop, Darmstadt, Germany, pp. 1-8, September 29, 2002. http://dx.doi.org/10.1109/ART.2002.1106948

[13] A K.Sin and H B. Zaman, "Tangible Interaction in Learning Astronomy through Augmented Reality Book-Based Educational Tool," First International Visual Informatics Conference, IVIC 2009, Kuala Lumpur, Malaysia, pp. 302-313, November 11-13, 2009.

[14] G. Lee, A. Andreas, and A. Kim, "CityViewAR: A Mobile Outdoor AR Application for City Visualization," IEEE International Symposium on Mixed and Augmented Reality, Atlanta, Georgia, pp. 5-8, November, 2002.

[15] E. Akao, M. Endo, T. Naka, K, Iwazaki, K. Mouri, and T. Yasuda, "Design and Development of Tablet-based Digital Teaching Material for Annular Eclipse Lectures based on Cooperation with a Museum," 2012 The Society of Socio-Informatics, pp. 301-304, September 14-16, 2012.

[16] M. Ura, M. Endo, M. Yamada, S. Miyazaki, K. Iwazaki, K. Mouri, and T. Yasuda, "A Smartphone Application for Searching Constellations Interactivity," Workshop on Advanced Image Technology Technical Program, pp. 283-288, January 7-8, 2013.

[17] M. Jean, Astronomical Algorithm, pp. 59-60, 1998, WillmannBell.

[18] M. Barnett, "Using Virtual Reality Computer Models to Support Student Understanding of Astronomical Concepts," Journal of Computers in Mathematics and Science Teaching, vol. 24 no. 4, pp. 333-356, 2005.

\section{AUTHORS}

K. Tian is a Ph.D. candidate at the Graduate School of Information Science at Nagoya University, Japan. He can be reached at tianke0711@gmail.com.

M. Endo is an associate professor at the Graduate School of Information Science at Nagoya University, Japan. He can be reached at endo@is.nagoya-u.ac.jp.

M. Urata is an assistant professor at the Graduate School of International Development, Nagoya University, Japan. She can be reached at mayu@nagoya-u.jp.

K. Mouri is a curator of the planetarium at Nagoya City Science Museum, Japan. He can be reached at mouri@nagoya-p.jp.

T. Yasuda is a professor at the Graduate School of Information Science, Nagoya University, Japan. He can be reached at yasuda@is.nagoya-u.ac.jp.

Submitted, March 26, 2014. Published as resubmitted by the authors June 08,2014 